Supporting Information

\title{
Dual-Emissive Near-Infrared Carbon Dot-Based Ratiometric Fluorescence Sensor for Lysozyme
}

Wan $\mathrm{Ji}^{\dagger}$, Jialuo $\mathrm{Yu}^{\ddagger}$, Jianxia Cheng ${ }^{\dagger}$, Longwen $\mathrm{Fu}^{\ddagger}$, Zhiyang Zhang ${ }^{\ddagger}$, Bowei $\mathrm{Li}^{\ddagger}$, Lingxin Chen* ${ }^{* \dagger,}$, Xiaoyan Wang ${ }^{*, \dagger}$

$\dagger$ School of Pharmacy, Binzhou Medical University, Yantai 264003, China

* CAS Key Laboratory of Coastal Environmental Processes and Ecological Remediation, Shandong Key Laboratory of Coastal Environmental Processes, Research Centre for Coastal Environmental Engineering and Technology, Yantai Institute of Coastal Zone Research, Chinese Academy of Sciences, Yantai 264003, China

*Corresponding Authors

E-mail addresses: wangxy@yic.ac.cn (X. Wang); 1xchen@yic.ac.cn (L. Chen). 


\section{Contents}

Reagents and materials.

Instrumentation.

MTT assay for the cell cytotoxicity.

ELISA assay for LYZ.

Scheme S1. Schematic illustration for the mechanism of the nanosensor (I. electrostatic interaction, II. the change of LYZ microenvironment, III. the interaction between functional groups).

Fig S1. TEM and HRTEM images of the dNIR-CDs.

Fig S2. FT-IR spectrum of the dNIR-CDs.

Fig S3. XRD pattern of the dNIR-CDs.

Fig S4. (a) XPS survey spectrum, (b) C 1s XPS spectrum, (c) N 1s XPS spectrum, (d) S 2p XPS spectrum of dNIR-CDs.

Fig S5. The UV-vis spectra of dNIR-CDs in the absence (blue line) and presence (red line) of LYZ.

Fig S6. Fluorescence emission spectra of the dNIR-CDs under different excitation wavelengths.

Fig S7. (a) Fluorescence intensity changes of dNIR-CDs for $120 \mathrm{~min}$ under $400 \mathrm{~nm}$ excitation. And the effect of temperature (b) and ionic strengths (c) on the fluorescence intensity of dNIR-CDs.

Fig S8. Fluorescence emission decay curves of dNIR-CDs (blue line) and dNIR-CDs+LYZ (red line).

Fig S9. The circular dichroism spectra of LYZ with different concentrations of dNIR-CDs.

Fig S10. Effect of (a) time and (b) $\mathrm{pH}$ on the nanosensor performance for LYZ detection (fluorescence intensity at $680 \mathrm{~nm}$ ). The concentration of LYZ was $0.5 \mu \mathrm{M}$.

Fig S11. Cell viability assay (MTT) of HMC in the presence of NIR-CDs for $24 \mathrm{~h}$.

Table S1. The $\zeta$ potentials of dNIR-CDs, LYZ and their mixtures, respectively.

Table S2. Determination of intracellular LYZ using ELISA kit.

Table S3. Comparison of different reported fluorescence methods for LYZ detection. 
Reagents and materials. Lysozyme (LYZ) was purchased from Sigma-Aldrich (Shanghai, China). Glutathione $(\mathrm{GSH})$ and polyethylenimine (PEI, Mw=600) obtained from Macklin Biochemical Technology Co., Ltd. (Shanghai, China). Formamide, arginine (Arg), glucose (Gluc) and dimethylsulfoxide (DMSO) were purchased from Sinopharm Chemical Reagent Co., Ltd (Shanghai, China). Glycine (Gly) and serine (Ser) were purchased from Aladdin (Shanghai, China). BCA protein concentration Kit, RIPA Tissue/Cell Lysate Kit, Trypsin (Try), thrombase (THr), acetylcholinesterase (AChE), penicillin streptomycin and PBS buffer were all purchased from SolarBio Science \& Technology Co., Ltd. (Beijing, China). Immunoglobulin G (IgG) was produced by Dingguo Changsheng Biotechnology Co., Ltd. (Beijing, China). The cell medium used to culture cell was Dulbecco's modified Eagle medium (DMEM) from Cytiva Inc., USA. Fetal bovine serum (FBS) was purchased from Gibco (USA). Trypsin-EDTA (0.25\%) was manufactured by Shanghai Biyuntian Biotechnology Co., Ltd. All reagents were used directly without further purification. Water used in the all experiments was ultrapure water. Human mesangial cells (HMC) were purchased from the Committee on Type Culture Collection of Chinese Academy of Sciences (Shanghai, China). Lysozyme ELISA Kit was purchased from Wuhan Fine Biotech Co., Ltd.

Instrumentation. The Fluorescence Spectrophotometer (F-7000 Hitachi) was used to record fluorescence spectra. UV-visible spectra were obtained by Thermo Scientific Nanodrop 2000/2000C spectrophotometer (Thermo Fisher Scientific, Waltham, MA). $\zeta$ potential was detected on Malvern Zetasizer Nano-ZS90 (ZEN3590, UK). X-ray photoelectron spectroscopy (XPS) was measured by Escalab XI + spectrometer (Thermo Scientific, USA). A Fourier transform infrared spectrometer (Nicolet IS10, Thermo Fisher) was used for identification of functional groups of materials. Morphological evaluation was observed by transmission electron microscopy (TEM, JEM-1230, HRTEM, JEOL 2100F, operating at $100 \mathrm{kV}$ and $200 \mathrm{kV}$, respectively). The fluorescence lifetime was measured on the Edinburgh Instruments, FSL980. MTT Assay was carried out by a microplate reader (Tecan, Austria). XRD was performed in Smartlab 9, Rigaku. The circular dichroism spectra were obtained by Chirascan qCD.

MTT assay for the cell cytotoxicity. MTT assay is a quantizetatative colorimetric assay that estimates the in vitro cytotoxicity of dNIR-CDs using HMC [1]. $100 \mu \mathrm{L}$ of cells were seeded in a 96-well plate with a density of $6 \times 10^{5}$ cells per well. Six replicate wells were used for each control and tested concentrations. After incubation for $24 \mathrm{~h}$, cells were treated with another $100 \mu \mathrm{L}$ of DMEM, containing different concentrations of dNIR-CDs $(0-1 \mathrm{mg} / \mathrm{mL})$ for another $24 \mathrm{~h}$. Then, $10 \mu \mathrm{L}$ of MTT was added to each well. After additional $4 \mathrm{~h}$ incubation, the growth media were removed, and the obtained mixtures were dissolved in $100 \mu \mathrm{L}$ DMSO and shaken for $10 \mathrm{~min}$. Finally, the optical density of each sample was recorded using a microplate reader at a wavelength of $490 \mathrm{~nm}$. The cell viability was calculated according to the following equation:

$$
\text { Cell viability }(\%)=\mathrm{OD}_{\text {treated }} / \mathrm{OD}_{\text {control }} \times 100 \%
$$


Where $\mathrm{OD}_{\text {treated }}$ is the absorbance of the experiment group (in the presence of $\mathrm{dNIR-CDs}$ ) and $\mathrm{OD}_{\text {control }}$ is the absorbance of the control group (in the absence of dNIR-CDs).

ELISA assay for LYZ. ELISA kit was used to detect the intracellular LYZ. Human mesangial cells (HMC) were cultured overnight in a six-well plate. The experimental group was incubated with $2 \mu \mathrm{M} \mathrm{LYZ}$ for $30 \mathrm{~min}$, and the control group which cells without LYZ treatment. After adequate washing with PBS, the next experiment was carried out. The cells of these two groups were lysed using RIPA kit (SolarBio Science \& Technology Co., Ltd. (Beijing, China)), and the supernatant was collected by centrifugation to obtain cellular proteins. LYZ in cells was measured by lysozyme ELISA kit (Wuhan Fine Biotech Co., Ltd). The above experiments were carried out in strict accordance with the kit instructions.

Scheme S1. Schematic illustration for the mechanism of the nanosensor (I. electrostatic interaction, II. the change of LYZ microenvironment, III. the interaction between functional groups).
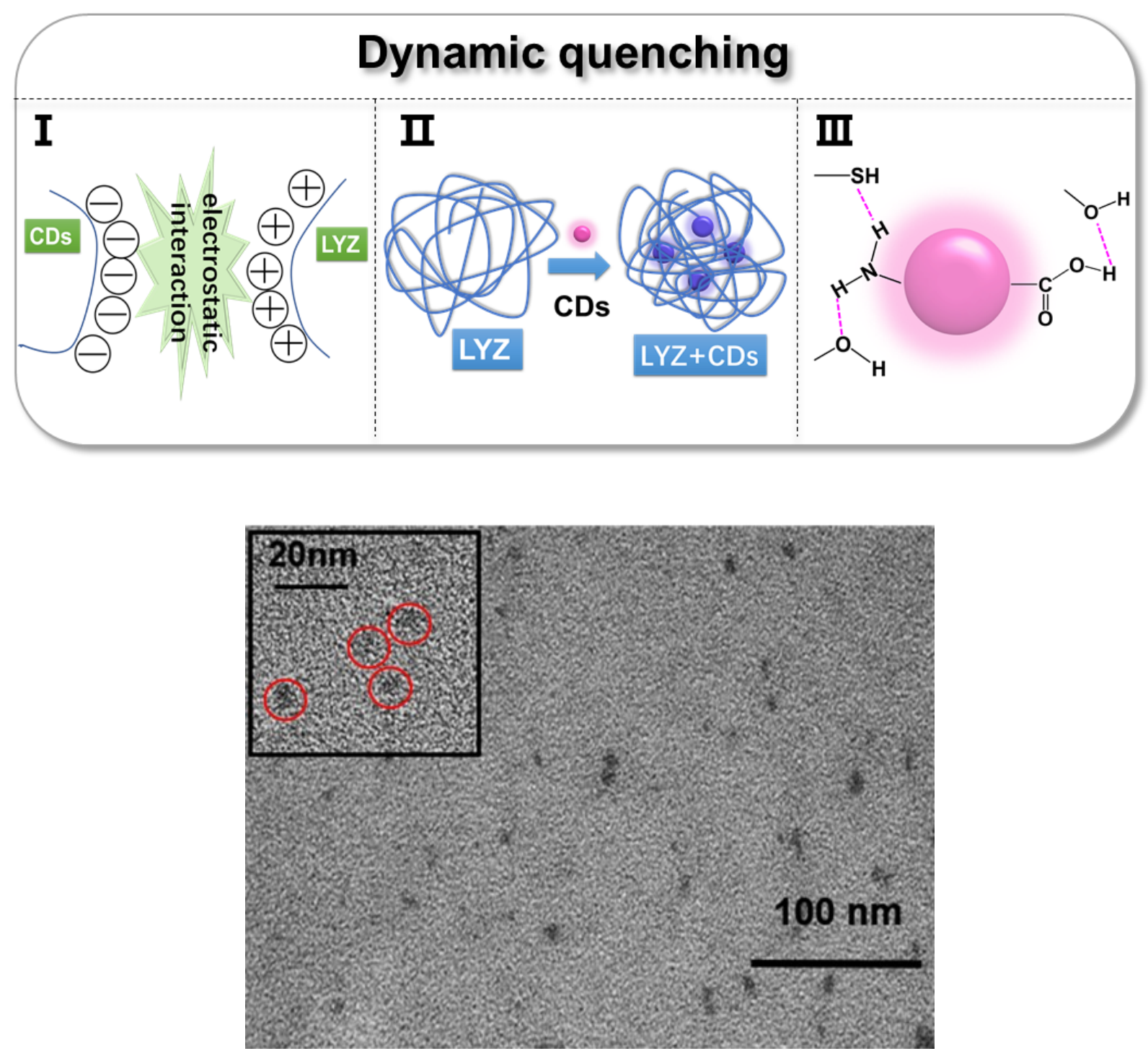
Fig S1. TEM and HRTEM (inset) image of the dNIR-CDs.

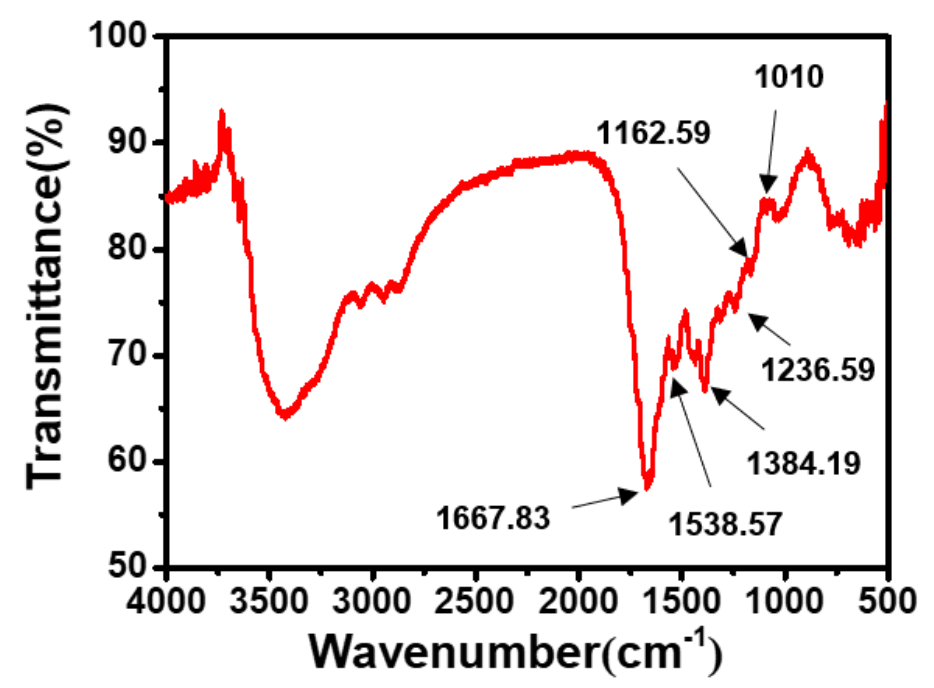

Fig S2. FT-IR spectrum of the dNIR-CDs.

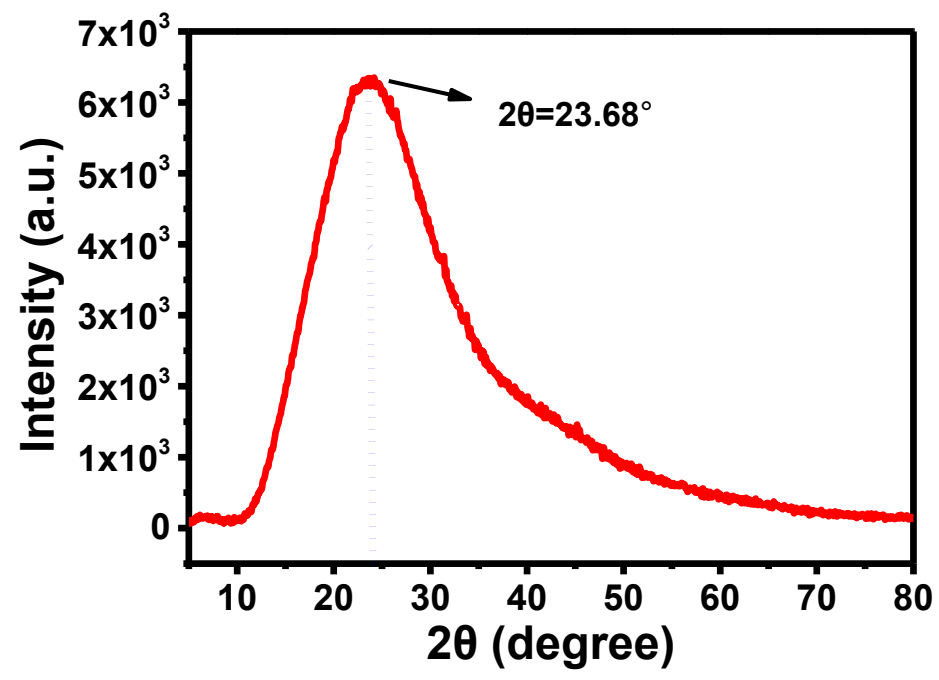

Fig S3. XRD pattern of the dNIR-CDs. 

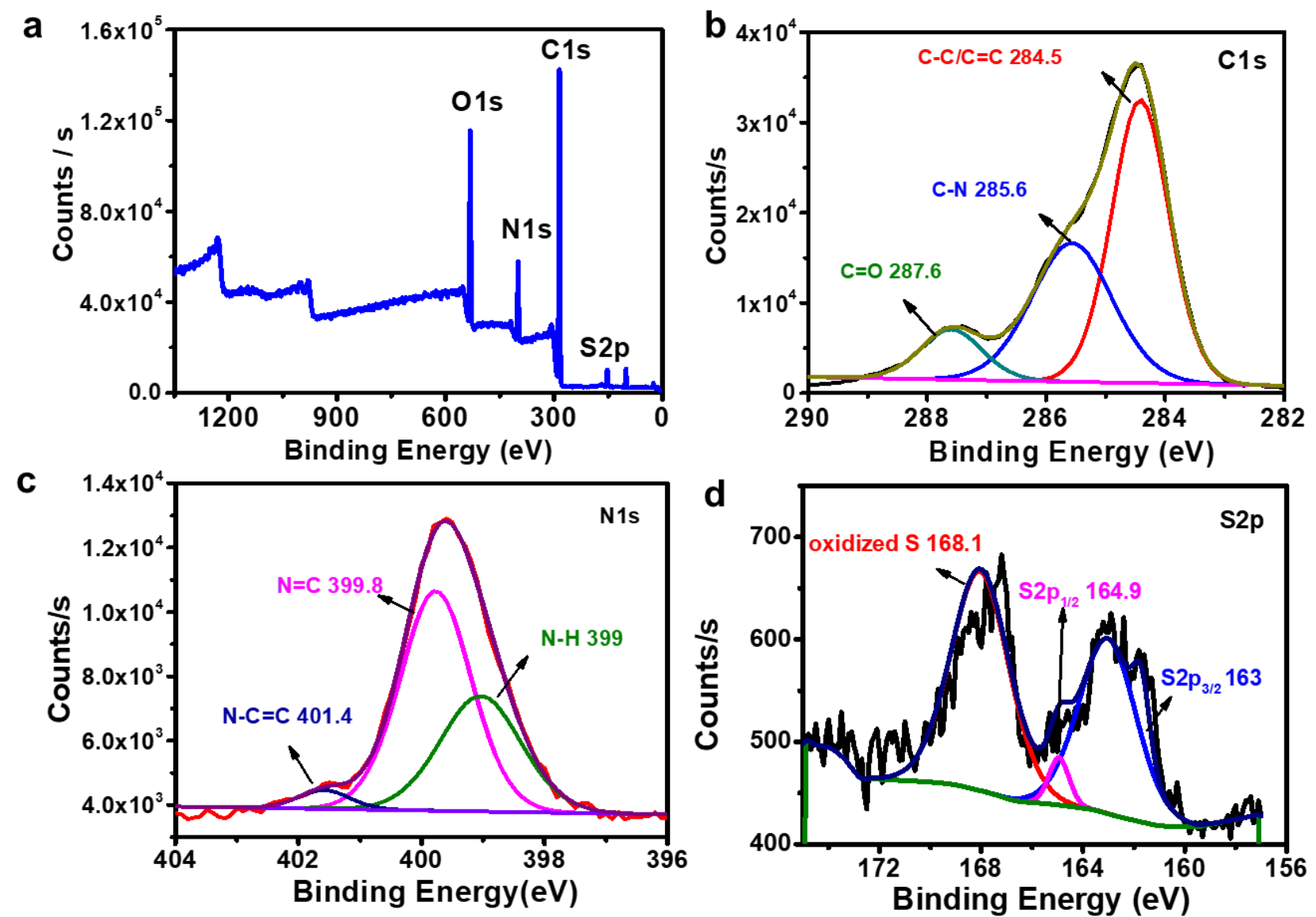

Fig S4. (a) XPS survey spectrum, (b) C 1s XPS spectrum, (c) N 1s XPS spectrum, (d) S 2p XPS spectrum of dNIR-CDs.

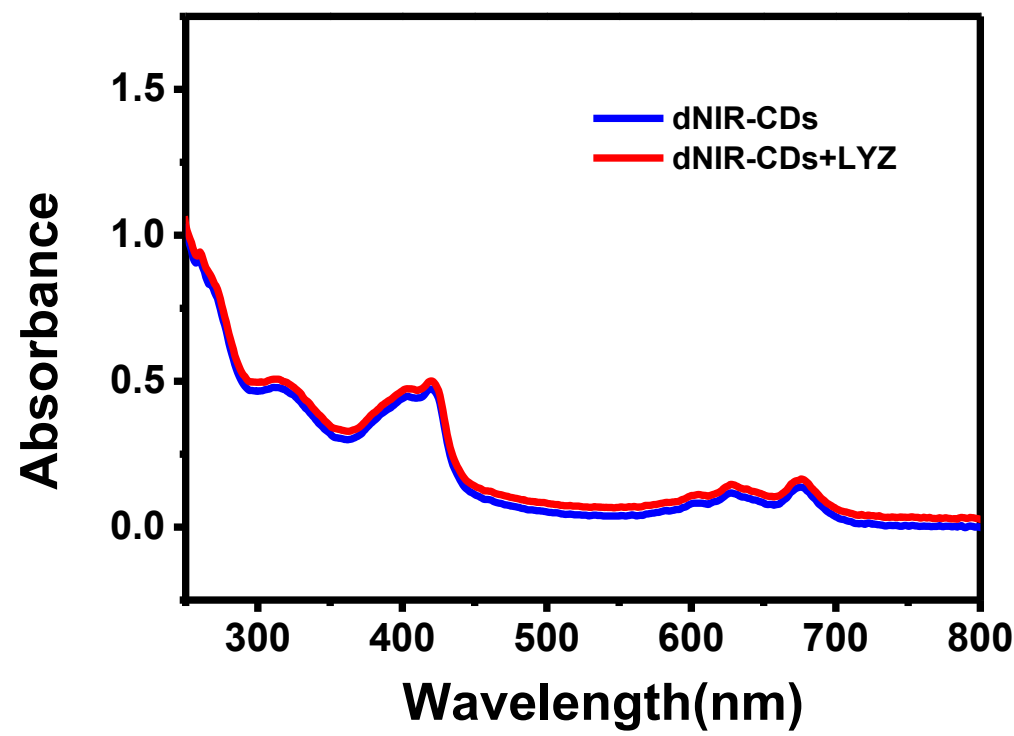

Fig S5. The UV-vis spectra of dNIR-CDs in the absence (blue line) and presence (red line) of LYZ. 


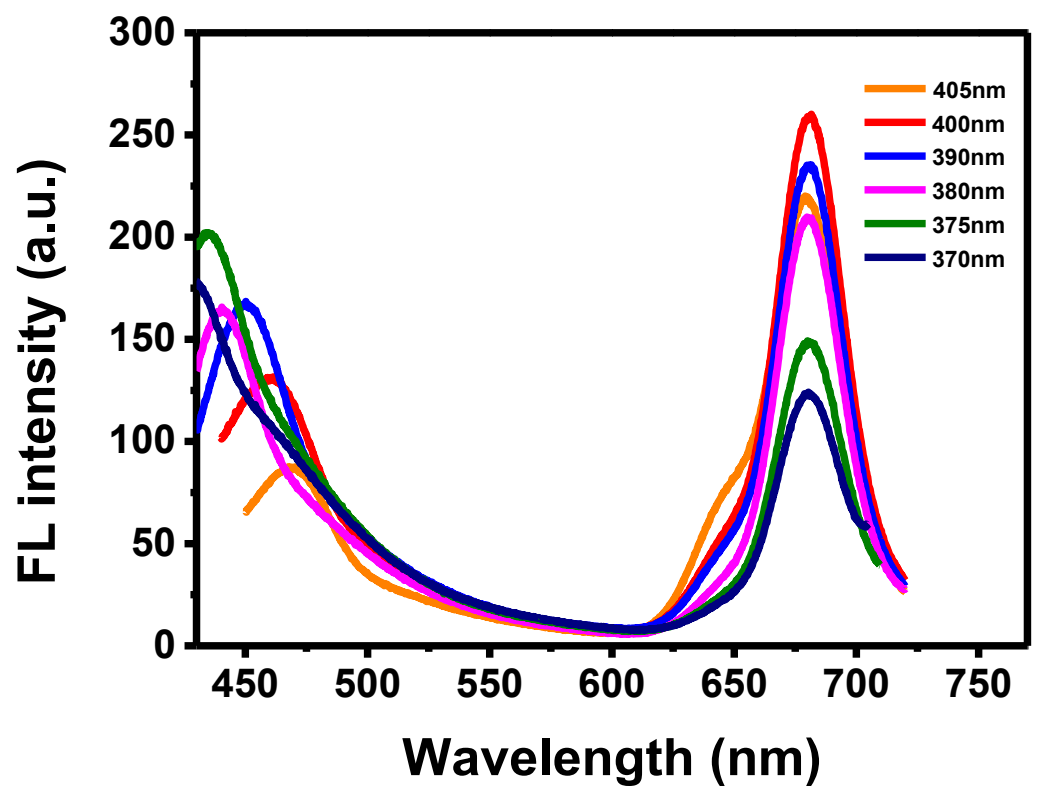

Fig S6. Fluorescence emission spectra of the dNIR-CDs under different excitation wavelengths.
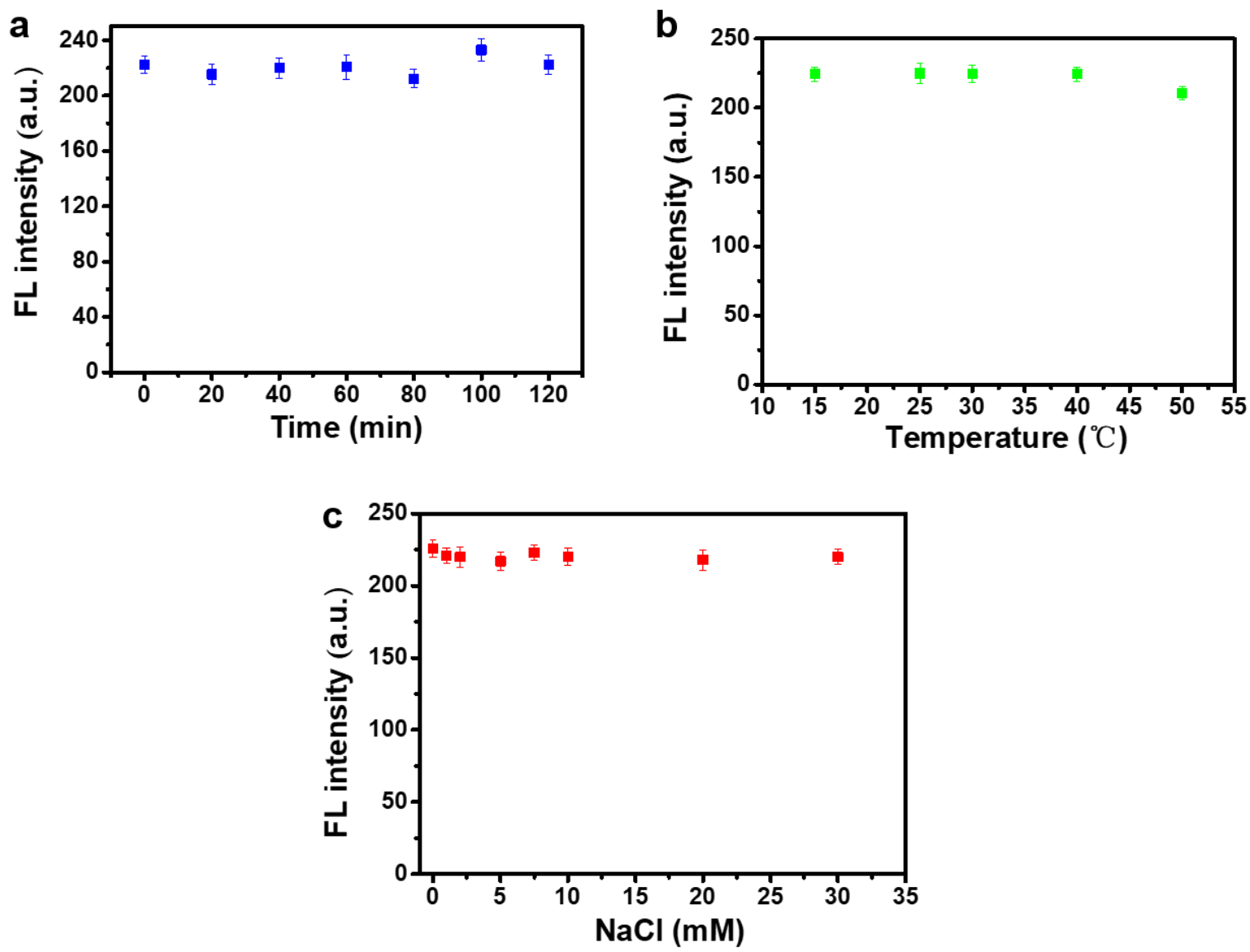

Fig S7. (a) Fluorescence intensity changes of dNIR-CDs for $120 \mathrm{~min}$ under $400 \mathrm{~nm}$ excitation. And the effect of temperature (b) and ionic strengths (c) on the fluorescence intensity of dNIR-CDs. 


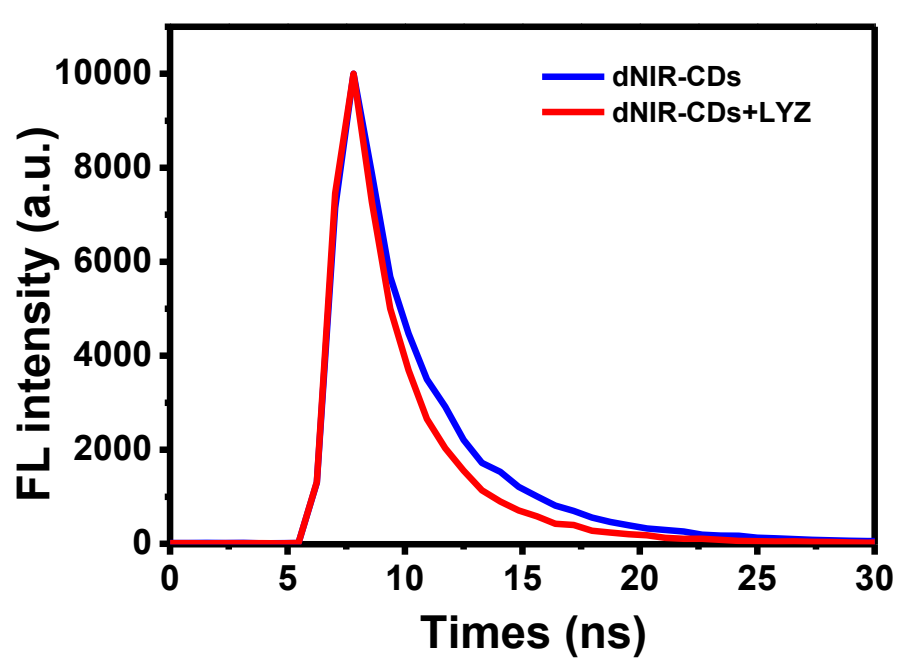

Fig S8. Fluorescence emission decay curves of dNIR-CDs (blue line) and dNIR-CDs+LYZ (10 $\mu \mathrm{M})$ (red line).

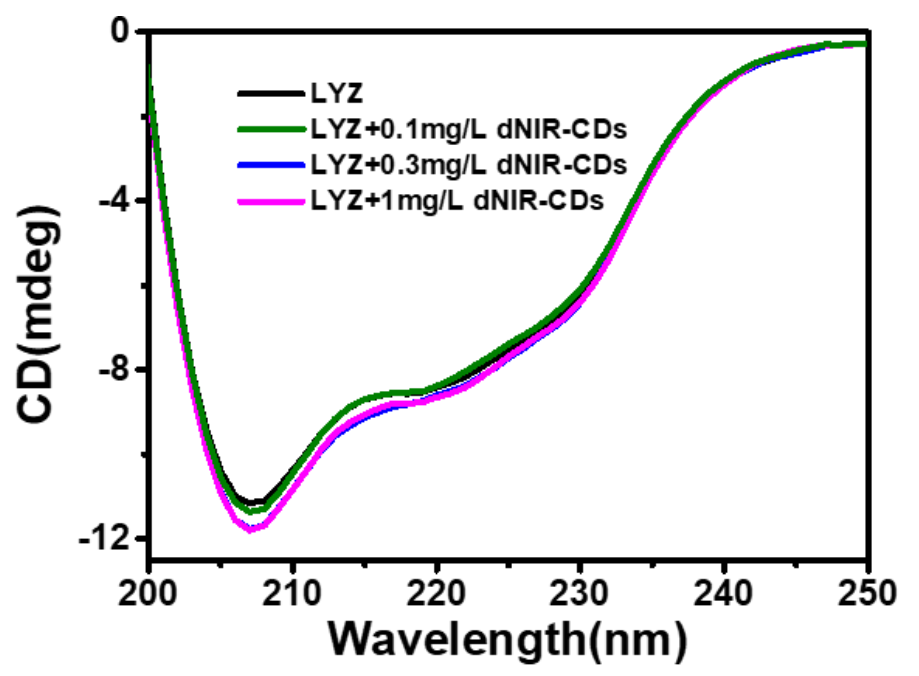

Fig S9. The circular dichroism spectra of LYZ with different concentrations of dNIR-CDs.
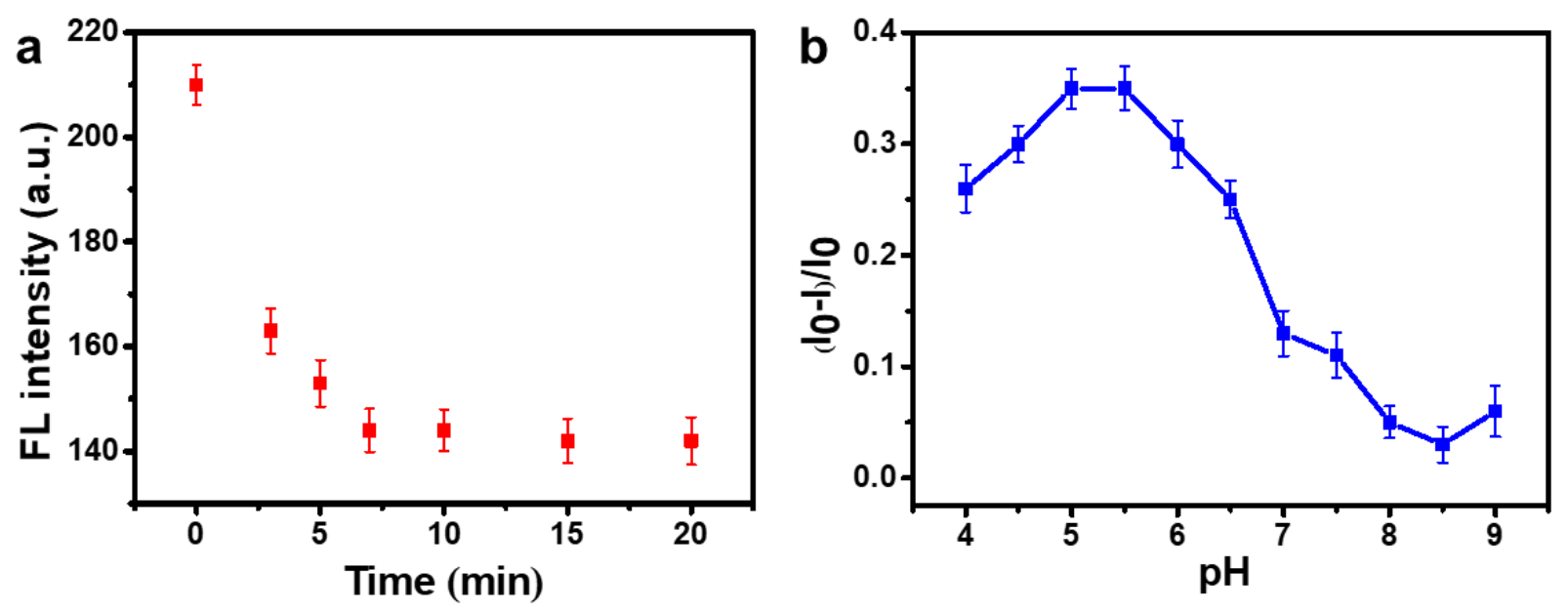

Fig S10. Effect of (a) time and (b) $\mathrm{pH}$ on the nanosensor performance for LYZ detection (fluorescence intensity at $680 \mathrm{~nm}$ ). The concentration of LYZ was $0.5 \mu \mathrm{M}$. 


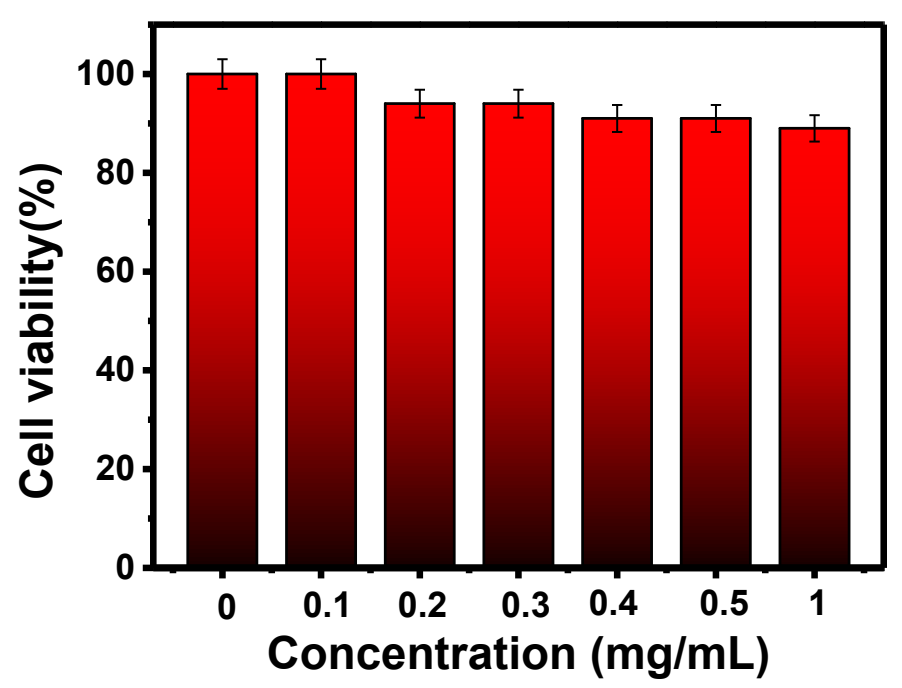

Fig S11. Cell viability assay (MTT) of HMC in the presence of dNIR-CDs for $24 \mathrm{~h}$.

Table S1. The $\zeta$ potentials of dNIR-CDs, LYZ and their mixtures, respectively.

\begin{tabular}{llll}
\hline & dNIR-CDs & LYZ & LYZ + dNIR-CDs \\
\hline$\zeta$ potential $(\mathbf{m V})$ & -8.91 & +12 & +7.08 \\
\hline
\end{tabular}

Table S2. Determination of intracellular LYZ using ELISA kit.

\begin{tabular}{lll}
\hline Samples & Concentration $(p g /(\boldsymbol{\mu g}$ protein $))$ & RSD $(\%)(\boldsymbol{n}=\mathbf{3})$ \\
\hline Control group & 0.42 & 2.5 \\
Experimental group & 2.58 & 2.2 \\
\hline
\end{tabular}


Table S3. Comparison of different reported fluorescence methods for LYZ detection.

\begin{tabular}{|c|c|c|c|c|c|c|}
\hline Recognition element & Method & $\begin{array}{c}\text { Emission peak } \\
(\mathrm{nm})\end{array}$ & Linear range & LOD & $\begin{array}{c}\text { Response time } \\
\text { (min) }\end{array}$ & References \\
\hline $\mathrm{CDs} / \mathrm{SiO}_{2} / \mathrm{MIP}^{a}$ & Fluorescence quenching & 420 & $70-700 \mathrm{nM}$ & $38 \mathrm{nM}$ & 60 & 49 \\
\hline $\mathrm{CdTe} / \mathrm{CdSe}$ & Fluorescence quenching & 645 & $5-500 \mathrm{nM}$ & $2.6 \mathrm{nM}$ & 70 & 50 \\
\hline \multicolumn{7}{|l|}{$\mathrm{QD}^{b}$-aptasensor } \\
\hline $\mathrm{CS}^{c}-\mathrm{GSH}-\mathrm{CuNCs}^{d}$ & Fluorescence quenching & 574 & $5-110 \mathrm{nM}$ & $1.6 \mathrm{nM}$ & 30 & 51 \\
\hline QD-embedded MIMs ${ }^{e}$ & Fluorescence quenching & 590 & $0.1-1 \mu \mathrm{M}$ & $10.2 \mathrm{nM}$ & 5 & 52 \\
\hline dNIR-CDs & Fluorescence ratiometric & 460 and 680 & $0.03-10 \mu \mathrm{M}$ & $7 \mathrm{nM}$ & 10 & This work \\
\hline
\end{tabular}

${ }^{a}$ Molecularly imprinted polymer. ${ }^{b}$ Quantum dot. ${ }^{c}$ Chitosan. ${ }^{d}$ Copper nanoclusters. ${ }^{e}$ Molecularly imprinted membrane.

\section{REFERENCE}

[1] Wang, M.; Kang, X.; Deng, L.; Wang, M.; Xia, Z.; Gao, D. Deep eutectic solvent assisted synthesis of carbon dots using Sophora flavescens Aiton modified with polyethyleneimine: Application in myricetin sensing and cell imaging. Food Chem. 2021, 345, 128817. 